\title{
Una propuesta de estrategias y recursos didácticos por competencias en respuesta a los estilos de enseñanza-aprendizaje de la población estudiantil ${ }^{1}$
}

\section{A Proposal of Didactic Strategies and Resources by Competencies in Response to Teaching- Learning Styles of the Student Population ${ }^{2}$}

\section{Uma proposta de estratégias didáticas por competências em resposta aos estilos de ensino-aprendizagem da população estudantil ${ }^{3}$}

Lizeth Valverde-López

Universidad Nacional

ROR:: https://ror.org/01t466c14

Heredia, Costa Rica

lizeth.valverde.lopez@una.cr https://orcid.org/0000-0002-4714-3776

Mariam Ureña-Hernández

Universidad Nacional

ROR:: https://ror.org/01t466c14

Heredia, Costa Rica

murenapz15@gmail.com

https://orcid.org/0000-0002-4100-2497

Recibido • Received • Recebido: 12 / 09 / 2019

Corregido • Revised • Revisado: 24 / 05 / 2021

Aceptado • Accepted • Aprovado: 05 / 07 / 2021

\begin{abstract}
Resumen
Objetivo. Diseñar estrategias y recursos didácticos por competencias para la asignatura de Administración de Documentos II de las carreras Administración de Oficinas y Educación Comercial impartidas por la Escuela de Secretariado Profesional. Metodología. El proceso se llevó a cabo con un enfoque cualitativo y por medio de los estudios descriptivo y exploratorio que permitieron conocer la opinión del estudiantado y personal académico; se utilizaron instrumentos semiestructurados para consultar sobre la predominancia de sistemas sensoriales, la aplicación de las estrategias y los
\end{abstract}

\footnotetext{
${ }^{1}$ Basado en el proyecto de graduación titulado Estrategias didácticas por competencias para el curso de Administración de Documentos II de la Escuela de Secretariado Profesional de la Universidad Nacional de Costa Rica en el II ciclo, 2017.

${ }^{2}$ Based on the graduation project titled Didactic strategies by competences for the course of Document Management II course of the Office Management and Commercial Education program at the National University of Costa Rica, Il cycle 2017.

${ }^{3}$ Com base no projeto de graduação intitulado Estratégias didáticas por competências para o curso de Administração de Documentos II da Escola de Secretariado Professional da Universidade Nacional da Costa Rica no Il ciclo, 2017.
} 
http://doi.org/10.15359/ree.25-3.7

http://www.una.ac.cr/educare

educare@una.ac.cr

recursos didácticos dentro del curso. Análisis de resultados. Los principales hallazgos demuestran que la población estudiantil que matricula el curso se encuentra entre los 19 y 24 años con una predominancia de los sistemas sensoriales: visual y kinestésico. Se resalta que los estilos de enseñanza integrador y laissez-faire, así como los estilos de aprendizaje acomodador y pragmático son los dominantes en esta población. Además, dentro de los recursos didácticos recomendados por el estudiantado, se encuentra el uso de la tecnología y la implementación de giras y visitas educativas, al igual que la resolución de casos y problemas. Conclusiones. Se manifiesta el requerimiento de la población estudiantil por aprender a hacer, formular y solucionar actividades, por eso se plantean estrategias didácticas que propicien las competencias. Se espera que esta investigación sirva de soporte para la labor docente y, a su vez, permita desarrollar una gestión encaminada a las necesidades educativas de cada dicente.

Palabras claves: Competencias; estilos de aprendizaje; estilos de enseñanza; estrategias didácticas; recursos didácticos; sistemas sensoriales.

\begin{abstract}
:
Objective. Design strategies and didactic resources by competencies for the Document Management II course of the Office Management and Commercial Education program at the Professional Secretariat School, National University of Costa Rica. Method. The process was carried out under a qualitative approach and through descriptive and exploratory studies that allowed knowing the students and academic staff's opinions. Semi-structured instruments were used to consult about the predominance of sensory systems, the application of strategies, and teaching resources within the course. Results. The main findings show that the student population enrolled in the course is aged 19 to 24 , with a predominance of visual and kinesthetic sensory systems. It is emphasized that the assimilating and laissez-faire teaching styles and the accommodator and pragmatic learning styles are the dominant ones in this population. In addition, the use of technology, the implementation of tours and educational visits, and the resolution of cases and problems are within the recommended teaching resources for this population. Conclusions. The student population's requirement to learn "how to do, formulate, and solve" activities is evident. Therefore, didactic strategies promoting competencies are proposed. It is expected that this research will serve as support for the teaching work and, in turn, will allow the development of management aimed at the educational needs of each student.
\end{abstract}

Keywords: Competence; teaching styles; learning styles; didactic strategies; didactic resources; sensory systems.

\title{
Resumo
}

Objetivo. Conceber estratégias e recursos didáticos por competências para a disciplina Administração de Documentos II dos cursos de Administração de escritorio e Educação Comercial ministrados pela Escola de Secretariado Professional. Metodologia. O processo foi realizado sob uma abordagem qualitativa e através de estudos descritivos e exploratórios que permitiram conhecer a opinião de estudantes e da equipe acadêmica; foram utilizados instrumentos semiestruturados para consultar sobre a predominância de sistemas sensoriais, a aplicação de estratégias e recursos de ensino no curso. Resultados. As principais descobertas mostram que a população estudantil que ingressa no curso tem entre 19 e 24 anos, com predominância de sistemas sensoriais: visual e cinestésico. 
http://doi.org/10.15359/ree.25-3.7

\begin{abstract}
Enfatiza-se que os estilos de ensino integrativo e deixar fazer, bem como os estilos de aprendizagem pragmático e de acomodação são os dominantes nessa população. Além disso, dentro dos recursos de ensino recomendados de população estudantil está o uso da tecnologia e a implementação de passeios e visitas educacionais, bem como a resolução de casos e problemas. Conclusão. Expressase a exigência da população estudantil de aprender a fazer, formular e resolver atividades, é por isso que as estratégias didáticas oferecidas são as que propiciam as competências. Espera-se que esta pesquisa sirva de apoio ao trabalho docente e, por sua vez, permita o desenvolvimento de uma gestão voltada para as necessidades educacionais de cada estudante.
\end{abstract}

Palavras-chave: Competências; estilos de ensino; estilos de aprendizagem; estratégias didáticas; recursos didáticos; sistemas sensoriais.

\title{
Introducción
}

El desafío primordial al que se enfrenta la educación superior es el fortalecimiento de las habilidades y destrezas de la población estudiantil, para obtener la adecuada interiorización de la información y, así, lograr una integralidad de los conocimientos con la práctica. Para alcanzar una adecuada implementación de las competencias en la educación universitaria, es necesario aplicar un método para el fortalecimiento del aprendizaje, de modo que se puedan combinar los conocimientos intrínsecos de cada estudiante y los nuevos aprendizajes que se desarrollan dentro del aula, con el fin de crear las bases necesarias para desempeñarse dentro del ámbito profesional.

Con base en lo anterior, se busca fortalecer las habilidades, aptitudes y destrezas en la población estudiantil, para lo cual se proponen estrategias didácticas por competencias para el Curso de Administración de Documentos II, cuya finalidad es que el estudiantado sea partícipe, propulsor y constructor del conocimiento, de manera que pueda ejecutar eficazmente las tareas asignadas en los entornos laborales y sociales.

Por lo tanto, el desarrollo de las estrategias didácticas por competencias para el curso de Administración de Documentos II se centra en la implementación de metodologías innovadoras que permitan alcanzar las competencias laborales requeridas en esta área por las carreras Administración de Oficinas y Educación Comercial de la Escuela de Secretariado Profesional (ESP) de la Universidad Nacional (UNA) de Costa Rica.

Para lograr este cometido, se realizó una investigación en el entorno internacional, lo que evidenció estudios como los de Alonzo Rivera y Valencia Gutiérrez (2016), Núñez Pérez (2013); Estrada García (2016) sobre las estrategias didácticas por competencias y el enfoque por competencias para distintas áreas; sin embargo, se carece de un estudio dirigido al curso Administración de Documentos II y que responda al diseño de estrategias didácticas que permitan el adecuado desarrollo y aplicación tanto teórica como práctica de los contenidos, lo que también amerita que el personal docente propicie un espacio educativo de construcción e integración del conocimiento. 
http://doi.org/10.15359/ree.25-3.7

http://www.una.ac.cr/educare

educare@una.ac.cr

En adición, dentro del ámbito costarricense, García Retana (2011), Araya Muñoz (2012), España Chavarría y Corrales Escalante (2014) han realizado estudios sobre las estrategias didácticas y las competencias donde concluyen que es necesaria la puesta en marcha de un cambio curricular del modelo tradicional hacia una educación orientada a las competencias, que potencie tanto las individuales como las colectivas; que es necesaria la integración de las tecnologías de información y comunicación dentro de los procesos educativos, así como la actualización del personal docente y el contenido de las clases para que se dé respuesta a las competencias digitales.

Además, personas autoras como Chaverría Cedeño y Ugalde Arguedas (2014) indican que el personal docente debe adaptar el programa y contenidos al contexto en el que se desenvuelve el estudiantado, por medio de la modificación de los recursos y estrategias didácticas.

\section{Referentes conceptuales}

Las competencias educativas procuran el desarrollo de las habilidades y destrezas propias de cada individuo, con la finalidad de que se pueda afrontar a las situaciones que se presentan en su entorno diario, tanto en el ámbito laboral, social o personal, de ahí que las competencias son definidas como "el conjunto de capacidades que se desarrollan mediante procesos, para que las personas sean competentes en múltiples aspectos" (Torres Rivera et al., 2014, p. 134). En este sentido, es requerido considerar que existen competencias tanto generales como específicas, las primeras hacen referencia a aspectos amplios que se pueden aplicar a distintas áreas del saber y según la profesión se deben ahondar, en cuanto a las segundas, hacen alusión a competencias de un perfil profesional específico, lo cual le da identidad (Torra et al., 2012).

En ese contexto, para lo requerido en el campo de la administración y gestión documental, es fundamental considerar los enfoques: constructivista, cognitivo y genérico que pueden permitir el desarrollo de las competencias específicas del perfil del curso.

El enfoque constructivista se reconoce como la implementación de las metodologías que permiten la construcción de los conocimientos por parte de los participantes del ámbito educativo, instaurando una perspectiva basada en la globalización del saber, compartiéndolo democráticamente y permitiendo que todos los individuos participen. Además, el constructivismo, según Arguedas Quesada (2014), utiliza la interacción del estudiantado con el objeto de estudio para lograr la apropiación del conocimiento.

En el enfoque cognitivo, el pensamiento humano juega un papel fundamental, debido a que se basa en la interrelación de los conocimientos previos con los nuevos; Serrano GonzálezTejero y Pons Parra (2011) indican que nace del supuesto de que la mente del ser humano es un sistema que opera con símbolos, de manera que la información es introducida, se codifica y parte de ella se almacena para ser consultada en el momento que sea requerido. En cuanto al enfoque genérico, combina elementos de las competencias como el qué hacer y cómo hacerlo, 
http://doi.org/10.15359/ree.25-3.7

en relación con las herramientas que se disponen para que el sujeto resuelva y se enfrente los retos que presentan los modelos educativos, ya que la finalidad de este enfoque es "identificar las posibles habilidades comunes que explican la diferencia entre un desempeño exitoso" (Tacca Huamán, 2011, p. 168) y uno deficiente.

Si bien es cierto, la mente del ser humano opera con símbolos y requiere captar la información a través de sus cinco sentidos, en donde cada uno de ellos tiene diferentes métodos para recibir y almacenar los datos; la combinación de estos sentidos permite el desarrollo de la capacidad de razonamiento a la persona; sin embargo, cada individuo tiene un canal fuerte, es decir, aunque todos los canales se encarguen de seleccionar la información, la persona capta y asimila de mejor manera mediante el sistema de experiencia sensorial dominante, el cual puede ser según la construcción desarrollada mediante el cuestionario VARK de Neil Fleming: visual, auditivo, lectoescritor y kinestésico (ver Figura 1) (León-Castañeda et al., 2010, citados por Gómez Bustamante et al., 2017).

Figura 1: Representación gráfica de los sistemas sensoriales
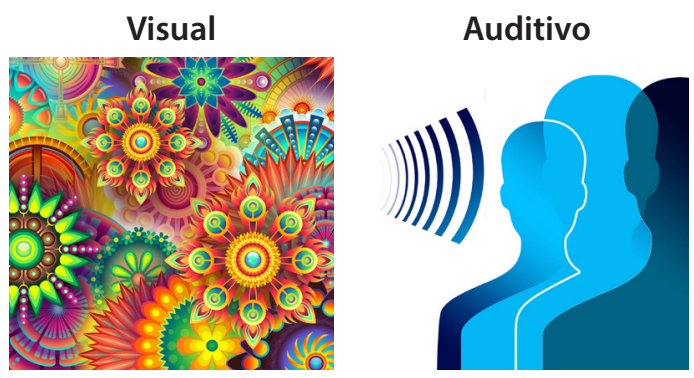

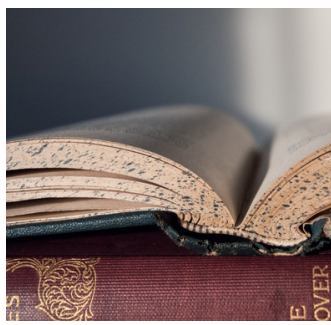

Lectoescritura

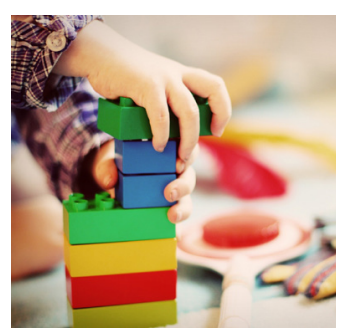

Kinestésico

Nota: Elaboración propia con imágenes tomadas de Pixabay.com

El sistema sensorial visual capta la información por medio de la vista, utilizando imágenes y representaciones gráficas para el establecimiento de relaciones entre distintas ideas; el auditivo, por su parte, se lleva a cabo mediante la escucha por medio del canal auditivo del oído, debido a esto facilita el aprendizaje por medio de la estimulación de distintos sonidos. En cuanto al lectoescritor, combina la escucha con la vista, a raíz de que se percibe mediante 
http://doi.org/10.15359/ree.25-3.7

http://www.una.ac.cr/educare

educare@una.ac.cr

la información expuesta en palabras escritas al ser leídas por la persona, a diferencia el sistema kinestésico se basa en el aprender haciendo. La autora Northon-Gámiz (2011) menciona que este sistema se evidencia cuando el estudiantado procesa la información según sus sensaciones y movimientos, por lo que la enseñanza y el aprendizaje orientados a las competencias buscan integrar distintas metodologías, estrategias y recursos que consideren las características y necesidades del estudiantado dentro del aula, ya que formar individuos competentes exige integrar estos aspectos y conocer a fondo la población con la que se trabaja.

Ahora bien, existen distintos estilos de enseñanza y de aprendizaje. Se entiende que los medios que utiliza el docente para facilitar la transmisión del conocimiento son los estilos de enseñanza y el proceso que se lleva a cabo durante la interacción del sujeto, sus características y la forma de aprender se conoce como los estilos o estrategias de aprendizaje. Con base en esto, para el desarrollo de las competencias se analizan los siguientes estilos de enseñanza y aprendizaje mediante una correlación, es decir, la compatibilidad entre el estilo de enseñanza que utiliza el personal docente con el estilo de aprendizaje que puede ser predominante en el estudiantado, debido a que al establecer estas correlaciones se potencia su efecto positivo dentro del aula.

Figura 2: Correlación entre los estilos de enseñanza y aprendizaje

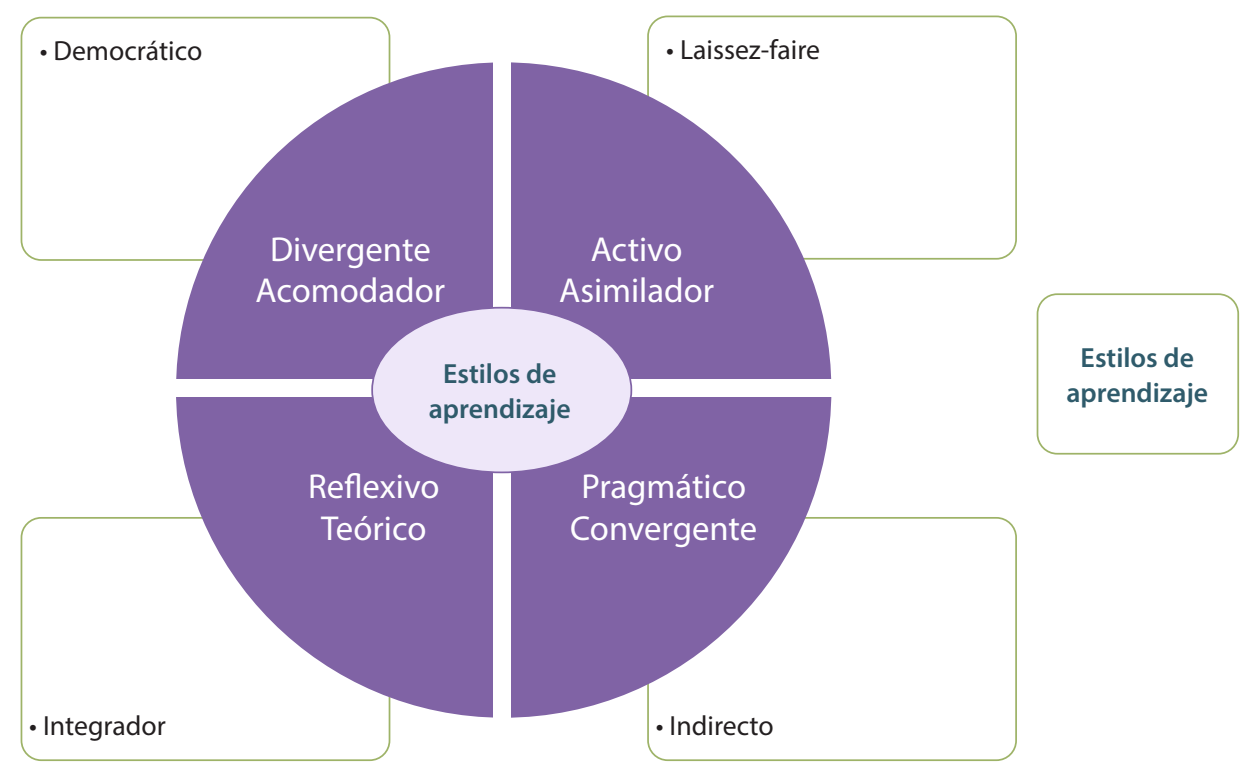

Nota: Elaboración propia con base en la clasificación de estilos de enseñanza de García et al. (2013) y de los estilos de aprendizaje por Honey y Mumford (1986) y McCarthy (1990) citados por Lozano Rodríguez y Tijerina Salas (2013) concuerdan con estas clasificaciones. 
http://doi.org/10.15359/ree.25-3.7

El estilo de enseñanza democrático se centra en el análisisy comprensión de las necesidades del estudiantado respecto a las temáticas de estudio, además de promover la autogestión por medio de la discusión, en consecuencia, es compatible y aplicable en conjunto con los estilos de aprendizaje divergente y acomodador, donde el primero promueve la habilidad imaginativa de cada persona, utilizando la formulación de lluvias de ideas para solucionar situaciones, y el segundo aplica la práctica de solución de casos y problemas para afrontar situaciones, por motivo de que se fundamenta en el aprender haciendo.

Por lo que respecta al estilo laissez-faire, promueve la iniciativa de los sujetos participantes al compartir el manejo y el planteamiento de la clase con el estudiantado; también beneficia la capacidad de retención de la información al tomar en cuenta los gustos y preferencias de cada individuo, a razón de esto se puede relacionar con los estilos de aprendizaje activo y asimilador, debido a que cada dicente puede ejecutar actividades de interiorización por medio de debates autodirigidos, métodos expositivos de temas de su interés como herramientas formadoras para el empoderamiento estudiantil dentro del aula.

En cuanto al estilo de enseñanza integrador y su relación con los estilos reflexivo y teórico (ver Figura 2), promueve las actividades cooperativas, el trabajo grupal y la solución de casos por medio de crítica constructiva y objetiva, características con mayor relevancia del estilo integrador; estas se pueden maximizar si se implementan acciones reflexivas de las temáticas por medio de la organización de ideas y conceptos, con la utilización, como principales herramientas, de la elaboración de mapas conceptuales, lluvias de ideas y diagramas de flujo. Además, la creación de hipótesis y comprobación de teorías a base del establecimiento de relaciones de las anécdotas con las temáticas de curso.

Por último, al plantear actividades que combinen lo vivido con los nuevos conocimientos se está aplicando el estilo de enseñanza indirecto, el cual tiene un mayor alcance si se ejecutan actividades demostrativas que sean realistas, es decir, que sean lo más parecido a la vida diaria y cercanas a las concepciones de vida de cada individuo, características de los estilos de aprendizaje pragmático y convergente.

Con base en estas clasificaciones de estilos de enseñanza y aprendizaje, es requerido conocer acerca de las actividades dentro del entorno educativo que se aplican con la finalidad de impartir, profundizar o ampliar contenidos, estas las estrategias didácticas que, además son conocidas como el sendero a seguir por el estudiantado para lograr una meta, es decir, las habilidades y destrezas que se pueden desarrollar (Orozco Alvarado, 2016) durante el proceso de enseñanza-aprendizaje. Para lograr el planteamiento de estrategias y actividades que propicien la adquisición de competencias educativas y laborales dentro del área de estudio, se analiza la aplicación de estrategias basadas en colaboración, problemas, proyectos e investigación. 
http://doi.org/10.15359/ree.25-3.7

http://www.una.ac.cr/educare

educare@una.ac.cr

La estrategia didáctica basada en colaboración es caracterizada por el trabajo en equipo, genera interacciones sociales que transforman conocimientos, de ahí que su importancia radica en que permite, al estudiantado, promover la comunicación y los ambientes de trabajo colaborativos que propician la inserción laboral por medio de la capacidad de autorregulación y tolerancia, además del desarrollo de competencias integradas tanto de carácter social como laboral.

En concordancia, la estrategia didáctica basada en problemas utiliza el trabajo en equipo, con la finalidad de sintetizar y construir el conocimiento mediante la resolución de problemas lo más parecidos a la realidad, para ayudar al estudiantado en la movilización de recursos, tanto conceptuales como procedimentales y actitudinales (Frola y Velásquez, 2011); por esto, la implementación de este tipo de estrategia se puede vincular con la aplicación de estrategias didácticas basadas en proyectos, debido a que su finalidad es acercar al estudiantado a la realidad del acontecer laboral y social, lo cual "permite poner en juego conocimientos, habilidades y actitudes a través del desarrollo de las actividades que desprenden de su planteamiento y realización" (Frola y Velásquez, 2011, p. 39).

En adición, la investigación permite que el estudiantado logre, de manera independiente, la búsqueda de información. Puebla Espinosa (2014) afirma que es un proceso de indagación sistemática sobre nuevos conocimientos, lo cual facilita a la población estudiantil el descubrir y buscar constantemente nueva información acerca de la realidad educativa que se vive. De igual manera, las estrategias didácticas basadas en casos plantean una herramienta para lograr que el estudiantado construya conocimientos, pero igualmente que trabaje de manera simultánea sus competencias por medio de ejercicios de resolución, ya que busca compensar la carencia de experiencia real (Millán, 1997, citado por López Cabral, 2014) que pueda presentar el estudiantado en áreas específicas de una profesión, debido a que se obtiene al experimentar la vida.

La aplicación de la estrategia didáctica en el aula se realiza por medio del recurso didáctico; a razón de esto, su implementación "queda completamente justificado cuando son integrados, de forma adecuada, en el proceso educativo, el cual debe ser compatible, a su vez, con el entorno más amplio que lo rodea (escolar, regional, social, etc.)"(Blanco Sánchez, 2012, p. 3), por esta razón son un apoyo para el proceso educativo, pues permiten la comunicación docente-estudiante y entorno físico-mental en relación directa con el desarrollo de los contenidos y material teórico.

En este sentido, las herramientas utilizadas desde tiempos remotos para complementar y ayudar en el proceso de aprendizaje son los recursos didácticos convencionales, cuyo resultado es la planificación docente para mediar en la clase, por lo cual, en primera instancia, se deben conocer las necesidades que presenta cada dicente; en segunda instancia, adecuar el recurso a los contenidos y las necesidades evidenciadas, para de esta manera lograr el desarrollo de las competencias en el área de estudio. Dentro de los recursos convencionales, Valle Pinta (2018) indica que se encuentran los materiales impresos, fotocopias, imágenes fijas, tableros didácticos, juegos, materiales de laboratorio, mapas conceptuales, organizadores, entre otros.

8 
http://doi.org/10.15359/ree.25-3.7

Mientras que los recursos que combinen lo auditivo con lo visual se conocen como audiovisuales y su finalidad es llamar la atención y ocupar la mente de las personas, algunos de los recursos audiovisuales de mayor acceso son, por ejemplo: la radio, la televisión, videos, el teléfono celular, reproductores MP4 y teleconferencias. Las tecnologías de la información y la comunicación (en adelante $\mathrm{TIC}$ ) pueden considerarse como recursos audiovisuales, ya que son herramientas desarrolladas por el ser humano para facilitar el conocimiento y, sobre todo, el alcance que se tiene de la información, de una manera más accesible y a bajo costo. Bautista Sánchez et al. (2014) aluden a que las TIC son una especie de unión entre la información, las telecomunicaciones y los medios audiovisuales; al respecto Monsalve Ochoa (2011) indica que en el momento que el estudiantado hace uso de las TIC se puede acoplar más fácilmente al mercado laboral, el cual solicita una persona competente en diversas áreas, una de esas, el uso de la tecnología. Asimismo, argumenta que muchas empresas brindan los servicios de manera virtual o mediante sistemas que ayudan al servicio del cliente; por último, expresa que el proceso de enseñanza y aprendizaje se puede ampliar con las oportunidades que ofrecen estos recursos tecnológicos.

\section{Metodología}

El paradigma permite delimitar las características que presentan la población y el sujeto de estudio en relación con el tema de investigación; tal como lo afirma Barrantes Echavarría (2014), el paradigma es la forma por la que se desarrolla la investigación, debido a que determina las acciones y el comportamiento de las comunidades sociales que se estudian. Por esto, el paradigma de esta investigación fue naturalista, a raíz de que abarca el conjunto de aspectos que intervienen en el problema y plantea un proceso de análisis y comprensión del entorno educativo y social actual con respecto de las necesidades formativas en el campo de estudio.

Se trabajó con un enfoque cualitativo, ya que tal como lo menciona Lerma González (2016), "interesa lo que la gente dice, piensa siente o hace" (p. 14), y con la aplicación de este enfoque, se buscaba conocer y comprender el entorno educativo, la conducta, características cognitivas y sociales de los participantes, así mismo las necesidades formativas desde su marco de referencia. En cuanto al tipo de estudio, se utilizó el descriptivo y el exploratorio, los cuales permitieron identificar y formular el problema de investigación, cuyos resultados, como recursos posteriormente, se podrían plantear por competencias, tomando como base los gustos, preferencias, estilos y métodos de aprendizaje de cada estudiante y acoplándolos a una estrategia que se pudiera aplicar de forma grupal.

En atención a lo anterior y para obtener la información necesaria, se aplicaron tres instrumentos: el cuestionario semiestructurado, la entrevista y el análisis del programa de estudio del curso, lo cual arrojó información de primera mano. El cuestionario semiestructurado se aplicó en el 2018 a 61 estudiantes de las carreras en investigación que habían cursado la materia Administración de Documentos II en el 2017. Cabe resaltar que la totalidad de personas 
http://doi.org/10.15359/ree.25-3.7

http://www.una.ac.cr/educare

educare@una.ac.cr

estudiantes que cursaron dicha materia en ese período fueron 87, por lo que el instrumento se aplica a más de la mitad. Con ello, el estudiantado tuvo la oportunidad de definir sus estilos y métodos de aprendizaje, gustos y preferencias educativas respecto a la naturaleza y modalidad en la que se imparte el curso, y brindó aportes a la metodología de enseñanza y recomendaciones sobre los materiales de apoyo que se podían utilizar.

Además, las personas estudiantes completaron el instrumento de validación de estrategias didácticas a finales de 2018, donde evaluaron las estrategias aplicadas mediante una escala de calificación que puntuaba cada criterio de la estrategia en escala de "Muy bien" (3), "Bien" (2) y "Puede mejorar" (1). Dentro de los criterios para evaluar el planteamiento de la estrategia se encontraban: si la estrategia era atractiva, amigable, fácil de utilizar; tenía instrucciones claras; permitía aplicar los conocimientos y contaba con un tiempo adecuado para su ejecución, entre otros específicos de la materia. Este fue un aporte significativo a la propuesta realizada como producto de la investigación.

Al personal docente que impartió el curso en 2017, tres académicos de la ESP, se les aplicó en 2018 una entrevista semiestructurada con preguntas abiertas y cerradas, en donde se abarcaron temas como el concepto de competencias, estrategias utilizadas para impartir los ejes temáticos y estilos de aprendizaje que predominaron durante el desarrollo del curso Administración de Documentos II. Las personas docentes a las que se le aplicó el instrumento son parte de la academia de la UNA y el país, han desempeñado labores docentes y administrativas en puestos de elección tanto dentro de la UNA como en el Ministerio de Educación Pública (MEP) de Costa Rica y el Colegio Universitario de Cartago (CUC).

Cabe resaltar que el personal docente también completó el instrumento de validación de las estrategias aplicadas. Los criterios de análisis consultaban sobre si la estrategia didáctica ejecutada se adecuaba al enfoque por competencias, a la temática del curso, a la infraestructura del lugar donde se imparte el curso; también evaluaba si permitía el desarrollo de competencias específicas como trabajo en equipo y bajo presión, entre otros aspectos relativos a la materia.

\section{Resultados}

El análisis de datos permite evidenciar características propias de la población con la que se trabaja en los cursos de Administración de Documentos II, por esta razón es necesario mencionar que el estudiantado se identifica dentro de la categoría de persona adulta joven, ya que se encuentra entre los 19 y 24 años, con predominancia mayoritariamente del sexo femenino. En este sentido, también es requerido conocer la experiencia con la que cuenta el personal docente que imparte los cursos actualmente, el cual se encuentra altamente calificado, con una experiencia superior a los ocho años dentro del sistema educativo universitario. 
http://doi.org/10.15359/ree.25-3.7

Ahora bien, el conocimiento de otras características de la población se vuelve indispensable para lograr el correcto planteamiento de estrategias y recursos didácticos por competencias dentro del área de estudio, la finalidad del estudio realizado; para ello se consulta al estudiantado y personal docente sobre la predominancia de sistemas sensoriales (ver Figura 3).

Figura 3: Sistemas sensoriales predominantes en la población estudiantil según la perspectiva estudiantil y docente

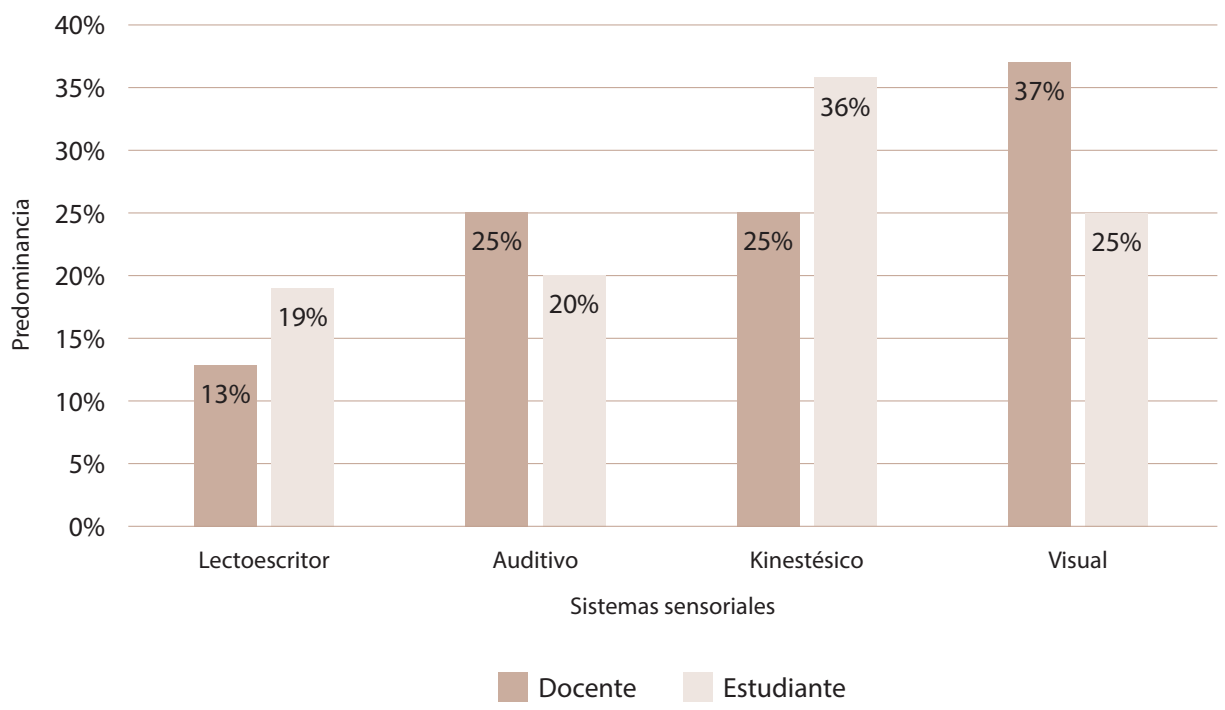

Nota: Elaboración propia.

Los sistemas sensoriales kinestésico y visual son los que predominaron, tanto desde la perspectiva del estudiantado como del personal docente, aunque el orden, tal como se observa en la Figura 3, es distinto. Resulta fundamental aprovechar las características que predominan en la población, por esto el planteamiento de recursos y estrategias didácticas por competencias debe ir orientado a la práctica del conocimiento empírico y considerar las habilidades blandas que permiten la construcción de nuevos juicios sobre el área temática.

Es primordial recordar que captar la información por medio del sistema visual implica la utilización de estrategias y recursos atractivos para la vista, con características como la armonía de colores y el movimiento, en concordancia, el sistema kinestésico se fundamenta en hacer, construir y reconstruir, para lograr esto, es necesario plantear actividades que despierten el interés de cada persona dentro del aula.

En relación directa con el conocimiento y la conciencia del sistema sensorial con mayor predominancia, también se deben conocer los estilos de enseñanza que predominan dentro 
http://doi.org/10.15359/ree.25-3.7

http://www.una.ac.cr/educare

educare@una.ac.cr

del entorno educativo (ver Figura 4), con la finalidad de realizar un planteamiento correcto de educación por competencias para esta área. Para obtener esta información se planteó la consulta tanto al personal docente como al estudiantado y se obtuvieron los siguientes datos.

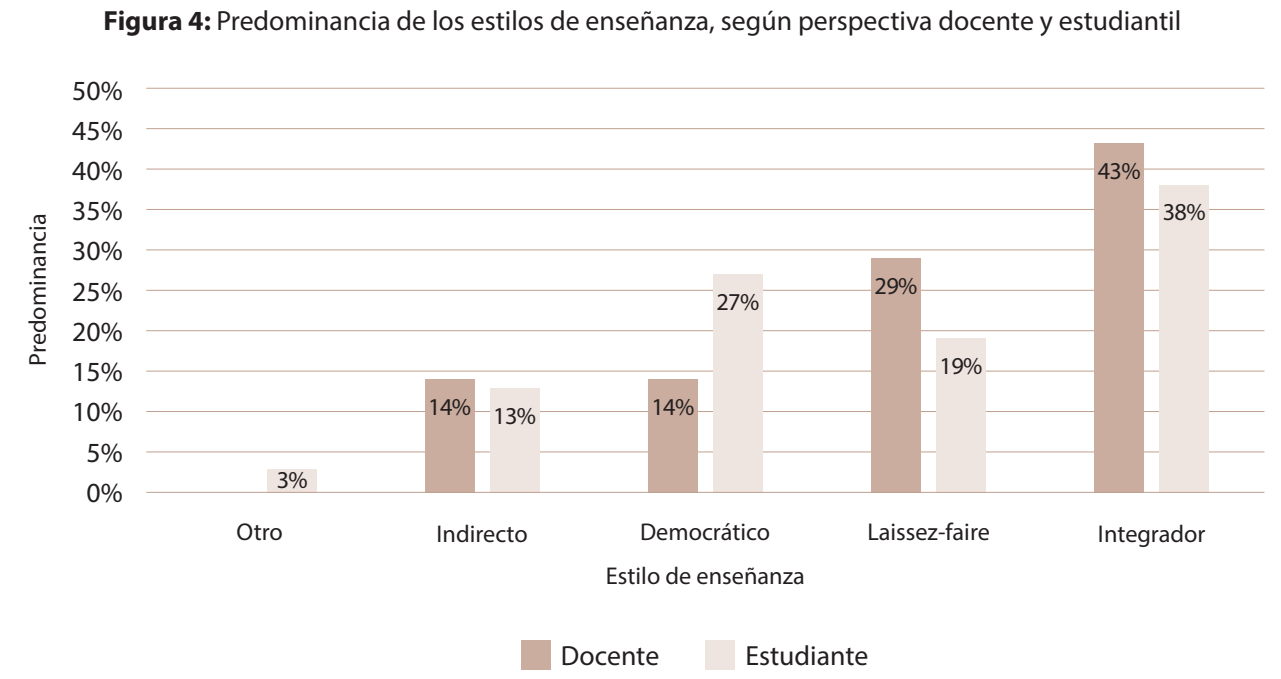

Nota: Elaboración propia a partir de los datos obtenidos

Inicialmente, se demuestra una predominancia del estilo de enseñanza integrador que se basa en el trabajo cooperativo encaminado a la crítica constructiva y objetiva para la resolución de casos, uno de los aliados las tecnologías de información y comunicación, lo cual permite aprovechar y maximizar el uso de los recursos tecnológicos dentro del aula. Seguidamente, se encuentran los estilos laissez-faire y democrático que buscan promover la iniciativa y autogestión durante los procesos educativos, tomando como base las necesidades de cada estudiante para formular distintas estrategias que permitan obtener la atención de la población estudiantil. Estas evidencias permiten delimitar el planteamiento de estrategias y recursos didácticos orientados a mantener activa a la persona estudiante promoviendo su capacidad crítica y buscando siempre su toma de decisiones.

En congruencia, los estilos de aprendizaje que predominaron en la muestra seleccionada son el pragmático y acomodador, lo cual refuerza el hallazgo de que se debe permitirse al estudiantado formular hipótesis y resolver las situaciones que se plantean. Se promueve el uso anécdotas y ejemplos de vida, así como la construcción de nuevo conocimiento a partir de estas experiencias, con la finalidad de que cada estudiante tenga un acercamiento lo más parecido a la realidad profesional y pueda aprender mediante el aprender haciendo, solucionando y resolviendo. 
http://doi.org/10.15359/ree.25-3.7

Para concretar el planteamiento de estrategias didácticas para el curso en cuestión y que respondan a las características específicas de la población estudiantil, se consultó sobre su preferencia de modalidad para matricular el curso (ver Figura 5), ya que en la UNA se imparten cursos presenciales, bimodales y virtuales.

Figura 5: Modalidad de matrícula del curso según la preferencia estudiantil

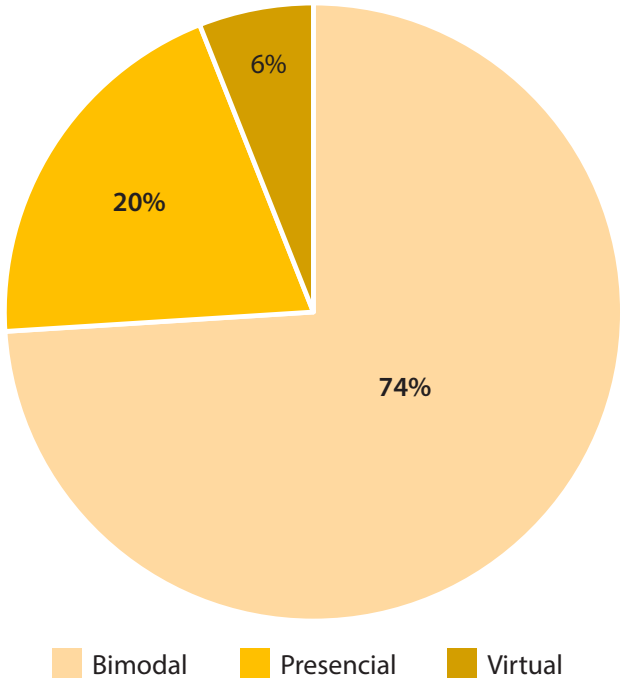

Nota: Elaboración propia a partir de la información obtenida.

La bimodalidad muestra predominancia, debido a que se ajusta a las necesidades educativas y personales del estudiantado al brindar la posibilidad de desarrollar actividades educativas desde la casa o conectados a internet sin el requerimiento de aproximarse presencialmente en algunas semanas, asimismo se tienen clases donde se requiere la asistencia del estudiantado al aula, lo cual manifiesta una ventaja para la población estudiantil, porque le permite evacuar dudas, ver y aprender nuevos contenidos.

De igual manera, esta modalidad exige que cada estudiante sea autodidacta, utilice la tecnología, nuevas aplicaciones y recursos, para lo cual debe tener disciplina con la entrega de los trabajos y poner en práctica competencias necesarias para enfrentar un mercado laboral exigente y demandante de resultados en cada tarea que se encomienda. Así también, se ajusta a la predominancia de los estilos de enseñanza y aprendizaje evidenciados en párrafos anteriores, lo que refuerza el planteamiento de estrategias y uso de recursos didácticos orientados a la construcción propia del conocimiento. 
http://doi.org/10.15359/ree.25-3.7

http://www.una.ac.cr/educare

educare@una.ac.cr

Tabla 1: Materiales de apoyo recomendados por la población estudiantil para su uso durante el curso Administración de Documentos II

\begin{tabular}{lr}
\hline \multicolumn{1}{c}{ Material de apoyo } & Porcentaje \\
\hline Apoyo tecnológico (uso de la computadora, el aula virtual, el internet y el programa de Excel & $36 \%$ \\
Mas prácticas y análisis de casos al utilizar un archivador real & $24 \%$ \\
Giras educativas o visitas a los archivos de las instituciones & $16 \%$ \\
Juegos individuales y colaborativos que permitan practicar la materia & $11 \%$ \\
Otros & $9 \%$ \\
Utilización de libros & $2 \%$ \\
Charlas & $2 \%$ \\
\hline Total & $100 \%$ \\
\hline
\end{tabular}

Nota: Elaboración propia con base en los datos obtenidos.

Respecto a los materiales y recursos de apoyo recomendados por la población estudiantil (ver Tabla 1), en primer lugar, se encuentran los recursos de apoyo tecnológico que fomentan la destreza, habilidad y conocimientos del estudiantado al estar expuestos a cambios constantes en aplicaciones, programas, métodos y herramientas de trabajo. En segundo lugar, se encuentran las prácticas y resoluciones de casos, que acercan al estudiantado a vivir problemas y situaciones que se pueden presentar en las empresas o instituciones, donde deben buscar alternativas para resolver estos casos, mediante el empleo de los recursos que se le brinden, para fomentar la eficiencia y eficacia.

En tercer lugar, se recomienda el planteamiento de las giras educativas, que son una muestra clara de la realidad laboral y acercan a cada estudiante al quehacer diario dentro de las instituciones; esto representa una oportunidad para que el estudiantado obtenga una noción clara sobre las actividades a las cuales se debe enfrentar en su día a día laboral como profesional en secretariado. En cuanto al material Otros, se refiere a distintos recursos como, por ejemplo, archivadores metálicos y de palanca, en adición a otros materiales básicos para el desarrollo de la administración documental. Para finalizar, el uso de libros y la implementación de charlas también fueron recomendados.

Por esto, tomando en cuenta los estilos de aprendizaje y los materiales de apoyo que sugiere el estudiantado, el grupo investigador plantea una serie de recursos y estrategias didácticas por competencias que faciliten la labor del personal docente del curso de Administración de Documentos II, al considerarse, de igual manera, la bimodalidad como preferencia del estudiantado para matricular el curso y ofrecer recursos que puedan ser ejecutadas en 
http://doi.org/10.15359/ree.25-3.7

medios informáticos de bajo costo, accesibles y fáciles de utilizar, como lo son las aplicaciones de Powtoon, Canva, Aula Virtual, Kahoot, Toondoo y Padlet; para esto también se proponen adaptaciones de juegos de mesa conocidos como el Jenga y las Cartas (ocho loco) que pueden brindar una opción para las clases presenciales.

Además, se recomienda, por parte del equipo investigador, la utilización de materiales reciclados para la resolución de casos y ejercicios a partir de hechos reales relacionados con los ejes temáticos del curso. Estos recursos plantean una alternativa que evita daños al ambiente y permite la práctica de los conocimientos adquiridos por medio de ejecución de actividades como rallys, giras, visitas y pasantías cortas a lugares de proyección laboral para las carreras.

\section{Consideraciones finales}

Con base en este análisis e investigación del entorno educativo, estilos de aprendizaje, estilos de enseñanza y otros datos que se pueden integrar durante el planteamiento de recursos y estrategias didácticas por competencias, se llega a las siguientes consideraciones.

Los estilos de aprendizaje con predominancia son los que exigen aprender a hacer, formular y solucionar actividades, dentro de las cuales resaltan las prácticas, casos y problemas que se encuentren profundamente ligados con la vida diaria, de ahí que las propuestas didácticas que se plantean a raíz de esta investigación tienen como fin último adecuarse a las necesidades estudiantiles, al fomentar un método lúdico, novedoso y divertido para poder desarrollar y ejecutar las competencias.

Es imperante ejecutar estrategias y recursos didácticos que consideren las herramientas tecnológicas que se encuentran al alcance, como lo son las aulas virtuales institucionales, el Facebook, las pizarras interactivas, el proyector multimedia y el computador, debido a que concuerdan con los sistemas sensoriales que predominan en la población, el kinestésico y visual, en donde el estudiante tiene la posibilidad moverse, agruparse, hacer y rehacer las diferentes propuestas que el personal docente le plantea para su resolución. En escenarios virtuales se propone el uso de juegos de trivia sobre la materia por medio de herramientas como Kahoot como estrategia formativa; las conferencias virtuales con personal del área documental; herramientas como Toondoo que permiten plantear resoluciones de casos mediante creación de historietas o cuentos que atraen visualmente al estudiantado, le permiten mantenerse ocupado y facilitan el desarrollo de su raciocinio.

En este sentido, el desarrollo de actividades lúdicas convencionales también plantea una oportunidad para la ejecución de competencias relacionadas con el trabajo colaborativo, resolución de casos y problemas, creación de hipótesis y trabajos investigativos; actividades todas que crean en el estudiantado la capacidad para desenvolverse profesionalmente en un ambiente laboral exigente. Se propone el uso de rallys de aplicación de conocimientos, juegos 
http://doi.org/10.15359/ree.25-3.7

http://www.una.ac.cr/educare

educare@una.ac.cr

de jenga o cartas que plantean la resolución de trivias, casos o resolución de ejercicios prácticos. Estas propuestas dan oportunidades para que el estudiantado exponga sus criterios personales y resuelva en conjunto situaciones de vida cotidiana.

Finalmente, los resultados del proceso demuestran la necesidad de las personas estudiantes por evidenciar un cambio de metodología, donde tengan la posibilidad de vivenciar, ejecutar y resolver situaciones que las acerquen a la vida laboral. Siendo esta la finalidad de las carreras en cuestión, que su estudiantado graduado sea capaz de demostrar las competencias adquiridas.

Este proceso de investigación recomienda realizar estrategias y recursos didácticos estandarizados para la impartición de cada materia, de ahí la relevancia de contar con personal coordinador de cátedra para que, al finalizar cada proceso lectivo, las poblaciones graduadas cuenten con un estándar básico de conocimientos y competencias en la misma área.

\section{Declaración de Material complementario}

Este artículo tiene disponible, como material complementario:

-Guía de estrategias didácticas para el curso de Administración de Documentos II de la Escuela de Secretariado Profesional, en el enlace: https://issuu.com/una393/docs/unidad did ctica_estrategias_y recursos did_cticos

-La versión preprint del artículo en https://doi.org/10.5281/zenodo.4041989

\section{Referencias}

Alonzo Rivera, D. L. y Valencia Gutiérrez, M. del C. (2016). Competencias y estrategias didácticas desde el enfoque socioformativo, Quaderns Digitals.net, (82), 28-38. http:// www.quadernsdigitals.net/index.php?accionMenu=hemeroteca.VisualizaArticulolU. visualiza\&articulo $i d=11430$

Araya Muñoz, I. (2012). Construyendo el perfil por competencias para el profesional en educación comercial. Revista Electrónica Educare, 16(3), 203-226. https://doi.org/10.15359/ree.16-3.12

Arguedas Quesada, C. (2014). Los programas especiales del SINEM, su modelo pedagógico, las necesidades e intereses de sus estudiantes y el enfoque de derechos humanos: Análisis y sugerencias. Revista Actualidades Investigativas en Educación, 14(2), 1-31. https://doi. org/10.15517/aie.v14i2.14742

Barrantes Echavarría, R. (2014). Investigación: Un camino al conocimiento. Un enfoque cuantitativo, cualitativo y mixto. EUNED. 
http://doi.org/10.15359/ree.25-3.7

Bautista Sánchez, M. G., Martínez Moreno, A. R. y Hiracheta Torres, R. (2014) El uso de material didáctico y las tecnologías de información y comunicación (TIC's) para mejorar el alcance académico. Ciencia y Tecnología, (14), 183-194. http://www.palermo.edu/ingenieria/ pdf2014/14/CyT_14_11.pdf

Blanco Sánchez, M. I. (2012) Recursos didácticos para fortalecer la enseñanza-aprendizaje de la economía: Aplicación a la unidad de trabajo "Participación de los trabajadores en la empresa" [Tesis de maestría]. Universidad de Valladolid. https://uvadoc.uva.es/ bitstream/10324/1391/1/TFM-E\%201.pdf

Chaverría Cedeño, B. y Ugalde Arguedas, K. (2014). Estrategias didácticas basadas es sistemas simbólicos para mejorar en los estudiantes de decimo nivel de un colegio nocturno las representaciones mentales acerca de los temas de código genético del programa de biología de la educación diversificada [Trabajo de Licenciatura]. Universidad Nacional Costa Rica.

España Chavarría, C. y Corrales Escalante, X. (2014). Un estudio exploratorio sobre el desarrollo de competencias digitales en la educación superior. Revista ABRA, 34(49), 1-25. https://doi. org/10.15359/abra.34-49.5

Estrada García, A. (2016). Estrategias didácticas bajo el enfoque de competencias: Aplicación del uso de herramientas de forma interactiva. Revista Iberoamericana para la Investigación y el Desarrollo Educativo, 6(12), 1-14. https://doi.org/10.23913/ride.v6i12.224

Frola, P. y Velásquez, J. (2011). Estrategias didácticas por competencias: Diseños eficientes de intervención pedagógica para la educación básica y media superior y superior. $\mathrm{CIECI}$. http://secgral4.edu.mx/documentos/planeacion argumentada/estrategias didacticas competencias.pdf

García Retana, J. Á. (2011) Modelo educativo basado en competencias: Importancia y necesidad. Revista Actualidades Investigativas en Educación, 11(3), 1-24. http://www.redalyc.org/ pdf/447/44722178014.pdf

García, D. M., Joaquín, M., Torres, P. y Vázquez, I. R. (2013) Estilos de enseñanza y las nuevas tecnologías en la educación. ReiDoCrea Revista Electrónica de Investigación Docencia Creativa, (2), 219-225. http://digibug.ugr.es/bitstream/handle/10481/27766/ReiDoCreaVol.2-Art.31-Garcia-Torres-Vazquez.pdf?sequence=1\&isAllowed=y

Gómez Bustamante, E. M., Jaimes Morales, J. del C. y Severiche Sierra, C. A. (2017). Estilos de aprendizajeenuniversitarios, modalidaddeeducaciónadistancia.RevistaVirtualUniversidad Católica del Norte, (50), 383-393. http://www.redalyc.org/pdf/1942/194250865021.pdf

Lerma González, H. D. (2016). Metodología de la investigación: Propuesta, anteproyecto y proyecto

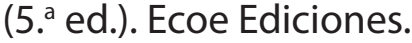


http://doi.org/10.15359/ree.25-3.7

http://www.una.ac.cr/educare

educare@una.ac.cr

López Cabral, S. (2014). El estudio de casos como estrategia de enseñanza y aprendizaje que promueven la educación científica. Congreso Iberoamericano de Ciencia, Tecnología, Innovación y Educación. https://docplayer.es/20864823-El-estudio-de-casos-comoestrategia-de-ensenanza-y-aprendizaje-que-promueven-la-educacion-cientifica.html

Lozano Rodríguez, A. y Tijerina Salas, A. (2013). La colaboración en espacios virtuales a través de estilos de aprendizaje desde la perspectiva docente: Un estudio de caso. Revista Estilos de Aprendizaje, 6(11), 38-50. http://revistaestilosdeaprendizaje.com/article/view/970

Monsalve Ochoa, M. (2011). Implementación de las TICs como estrategia didáctica para generar un aprendizaje significativo de los procesos celulares en los estudiantes de grado sexto de la institución educativa San Andrés del Municipio de Girardota [Tesis de maestría]. Universidad Nacional de Colombia. https://repositorio.unal.edu.co/handle/unal/9130

Northon-Gámiz, D. (2011) La detección de los estilos de aprendizaje. ¿Un objetivo o una necesidad? UCLM, Facultad de Ciencias Sociales de Cuenca. https://www.uclm.es/-/media/ Files/C01-Centros/cu-csociales/documentos2011/24-2011.ashx?la=es

Núñez Pérez, M. C. S. (2013). El docente en el enfoque por competencias. Pensamiento, Papeles de Filosofía, 1(1), 177-186. https://revistapensamiento.uaemex.mx/article/ download/340/314/

Orozco Alvarado, J. C. (2016). Estrategias didácticas y aprendizaje de las ciencias sociales. Ciencias de la Educación. Revista Científica de FAREM-Estelí. Medio ambiente, tecnología y desarrollo humano, (17), 65-80. https://doi.org/10.5377/farem.v0i17.2615

Puebla Espinosa, A. (3 de mayo, 2014). Importancia de la investigación educativa. Ponencia presentada en el Primer Congreso Internacional de Transformación Educativa. Consejo de transformación educativa. http://transformacion-educativa.com/index.php/articulossobre-educacion/54-importancia-de-la-investigacion-educativa

Serrano González-Tejero, J. M. y Pons Parra, R. M. (2011). El constructivismo hoy: Enfoques constructivistas en educación. Revista Electrónica de Investigación Educativa, 13(1), 1-27. http://dajimenez1.webs.com/UPN/El ConstructivismoHoy.pdf

Tacca Huamán, D. R. (2011). El "nuevo" enfoque pedagógico: Las competencias. Investigación Educativa, 15(28) 163-185. https://revistasinvestigacion.unmsm.edu.pe/index.php/educa/ article/view/5426

Torra, I., de Corral, I., Pérez, M. J., Triadó, X., Pagès, T., Valderrama, E., Dolors Màrquez, M., Sabaté, S., Solà, P., Hernàndez, C., Sagrà, A., Guàrdia, L., Estebanell, M., Patiño, J., González, À-P., 
http://doi.org/10.15359/ree.25-3.7 http://www.una.ac.cr/educare educare@una.ac.cr

Fandos, M., Ruiz, N., Iglesias, M. C. y Tena, A. (2012). Identificación de competencias docentes que orienten el desarrollo de planes de formación dirigidos a profesorado universitario. Revista de Docencia Universitaria. 10(2), 21-56. http://dugi-doc.udg.edu/ bitstream/handle/10256/10445//dentificacionCompetencias.pdf?sequence=1

Torres Rivera, A. D., Badillo Gaona, M., Valentin Kajatt, N. O. y Ramírez Martínez, E. T. (2014). Las competencias docentes: El desafío de la educación superior. Innovación Educativa, 14(66), 129-146. http://www.scielo.org.mx/pdf/ie/v14n66/v14n66a8.pdf

Valle Pinta, A. de J. (2018). Estrategias didácticas innovadoras en el aprendizaje del idioma inglés [Tesis de licenciatura]. Universidad Central del Ecuador. http://www.dspace.uce.edu.ec/ bitstream/25000/14499/1/T-UCE-0010-IN018-2018.pdf 